\title{
Inhibition of microtubule-associated protein 1 light chain 3B via small-interfering RNA or 3-methyladenine impairs hypoxia-induced HO8910PM and HO8910 epithelial ovarian cancer cell migration and invasion and is associated with RhoA and alterations of the actin cytoskeleton
}

\author{
ZHONGYUAN TANG ${ }^{1-3}$, NING ZHANG ${ }^{1}$, WEN DI $^{1,2}$ and WEIPING LI ${ }^{1,2}$ \\ ${ }^{1}$ Department of Obstetrics and Gynecology, Ren Ji Hospital, School of Medicine, Shanghai Jiao Tong University; \\ ${ }^{2}$ Shanghai Key Laboratory of Gynecologic Oncology; ${ }^{3}$ Department of Obstetrics and Gynecology, \\ Rui Jin Hospital, School of Medicine, Shanghai Jiao Tong University, Shanghai, P.R. China
}

Received November 25, 2014; Accepted December 22, 2014

DOI: $10.3892 /$ or.2015.3742

\begin{abstract}
Expression of microtubule-associated protein 1 light chain 3 (LC3) is correlated with poor prognosis in many human cancers. Hypoxia induces LC3 expression and is an essential characteristic of epithelial ovarian cancer (EOC). The aim of the present study was to elucidate the mechanism by which LC3 facilitates EOC cell migration and invasion under conditions of hypoxia. The effects of LC3B inhibition under hypoxic conditions on migration, invasion, and adhesion in HO8910PM and HO8910 EOC cell lines were investigated. LC3B inhibition was achieved by small-interfering RNA (siRNA) targeting LC3B or by treatment with 3-methyladenine (3-MA). Cell migration, invasion and adhesion and the arrangement of the cytoskeleton were determined by Transwell migration assays and rhodamine phalloidin staining. Western blot analysis was performed to evaluate the expression level of LC3B and the expression and activity of ras homolog gene family member A (RhoA). Increased LC3B expression was associated with HO8910PM and HO8910 cell migration and invasion promoted under hypoxic conditions. LC3B siRNA and 3-MA treatment each attenuated hypoxia-induced LC3B expression, along with migration and invasion, and this was associated with a decrease in RhoA expression and disorganization of the actin cytoskeleton. LC3B may promote the migration and invasion of EOC cells by affecting the cytoskeleton via the RhoA pathway. In addition, LC3B may be a marker of tumor hypoxia and/or metastasis in EOC cells.
\end{abstract}

Correspondence to: Dr Weiping Li, Department of Obstetrics and Gynecology, Ren Ji Hospital, School of Medicine, Shanghai Jiao Tong University, 1630 Dongfang Road, Shanghai 200127, P.R. China E-mail: liweiping_111@126.com

Key words: LC3B, hypoxia, epithelial ovarian carcinoma, RhoA, cytoskeleton

\section{Introduction}

Epithelial ovarian cancer (EOC) is the second most common gynecologic cancer and the most common cause of mortality from gynecologic malignancies. It is highly metastatic, and most patients are diagnosed at an advanced stage (1-3). Despite recent gains in survival, most patients experience recurrence and metastasis, and the 5-year survival rate for patients with advanced-stage ovarian cancer has remained unchanged (20-30\%) over the past 20 years $(4,5)$. Multiple genetic alterations are involved in the pathogenesis of ovarian cancer, and many of the signaling pathways and proteins involved have been characterized (6).

Metastasis is a complex process involving restructuring of the extracellular matrix and resulting in tumor cell migration, invasion, and establishment at new sites (7-9). Cell migration is a key factor in the invasion and metastasis of cancers (10). In addition, hypoxia is a common characteristic of growing solid tumors such as those of ovarian cancer $(11,12)$. Preliminary studies from our group have shown that drug resistance and metastasis are significantly enhanced in ovarian cancer cells under hypoxic conditions induced by physical or chemical methods (unpublished data).

Microtubule-associated protein 1 light chain 3 (LC3) is localized to the membrane bilayer of autophagosomes (13-16). It is converted from the cytosolic form (LC3-I) to the autophagosome membrane-associated form (LC3-II) and is degraded by lysosomal enzymes within autolysosomes during non-selective autophagy (17). LC3 was first identified as 1 of 3 light chains associated with purified microtubule-associated protein (MAP)1A and MAP1B and is considered to be involved in microtubule assembly/disassembly. It has been reported to bind to the Rab7 GTPase effector FYVE and coiled-coil domain 1 (FYCO1) to mediate microtubule plus-end-directed vesicle transport $(18,19)$. It has also been identified as a homolog of mammalian autophagy-related protein 8 (ATG8) (20).

LC3 expression has been reported to be common in the metastasis of various types of human cancer. Lazova et al (21) 
reported the common expression of LC3B in malignancies, supporting emerging evidence that autophagy plays a role in cancer progression. High levels of LC3B expression were associated with proliferation, invasion and metastasis, high nuclear grade, high mitotic rate, and worse outcome. Normal melanocytes adjacent to the tumor and melanoma cells of early in situ melanomas showed little or no LC3B expression. However, in primary invasive melanoma, punctate LC3B staining was widespread in nests of florid in situ tumor cells and in $100 \%$ of invasive tumors in the dermis (21). Sato et al (22) reported that $90 \%$ of primary colorectal tumors and $100 \%$ of lymph-node and liver metastases were positive for punctate LC3 expression. Normal mucosal epithelium adjacent to the tumors was negative. Yoshioka et al (23) reported strong LC3 expression in 53\% of esophageal, $58 \%$ of gastric, and $63 \%$ of colorectal cancer. Han et al (24) reported that overexpression of LC3 was associated with metastasis, poor clinical prognosis, and vasculogenic mimicry in patients with melanoma. Fujii et al (25) reported that $87 \%$ of their patients with pancreatic cancer were LC3B-positive, correlating with shorter disease-free periods and poor patient outcome.

The mechanism(s) underlying cytoskeletal changes and cell migration and invasion in EOC metastasis remains to be elucidated. The aim of the present study was to assess whether LC3 was involved in this process. We hypothesized that LC3 promotes tumor cell migration and invasion under hypoxic conditions by altering the cytoskeleton.

\section{Materials and methods}

Cell culture and reagents. Human HO8910PM and HO8910 EOC cell lines were obtained from the Chinese Academy of Sciences Cell Bank. The parental HO8910 cell line was established from ascites of a patient with malignant papillary serous adenocarcinoma of the ovary. HO8910PM is a highly metastatic clone obtained by limiting-dilution cloning of the HO8910 line and has been used in many studies, owing to its highly metastatic activity. Cells were grown in Roswell Park Memorial Institute (RPMI)-1640 medium (Thermo Scientific Hyclone, Logan, UT, USA) plus $10 \%$ fetal bovine serum (FBS; Gibco/Life Technologies, Carlsbad, CA, USA) at $37^{\circ} \mathrm{C}$ in a humidified atmosphere at normoxic conditions of $20 \% \mathrm{O}_{2}$, $5 \% \mathrm{CO}_{2}$ and $75 \% \mathrm{~N}_{2}$. Experiments were performed in 6-well culture plates at $80 \%$ cell confluence.

3-Methyladenine (3-MA), a specific inhibitor of autophagy and inhibitor of the conversion of LC3-I to LC3-II (26-29), was purchased from Sigma-Aldrich (St. Louis, MO, USA). Rabbit polyclonal anti-LC3B antibody (no. 2775) and rabbit monoclonal anti-Rho-associated, coiled-coil-containing protein kinase 1 (ROCK1) antibody (no. 4035) were purchased from Cell Signaling Technology (Beverly, MA, USA). Mouse monoclonal anti- $\beta$-actin antibody (mAb 8226) was purchased from Abcam (Cambridge, England, UK). Mouse monoclonal anti-ras homolog gene family, member A (RhoA) antibody (no. ARH03) and rhodamine phalloidin (no. PHDR1) were purchased from Cytoskeleton Inc. (Denver, CO, USA). The Rho Assay Reagent (Rhotekin RBD, agarose; recombinant protein expressed in Escherichia coli; no. 14-383, lot no. 2013105) was purchased from EMD Millipore (Billerica, MA, USA). Goat anti-rabbit IgG secondary antibody [AlexaFluor 488 goat anti-rabbit IgG (H+L); no. A11008] was purchased from Invitrogen/Life Technologies (Grand Island, NY, USA). Horseradish peroxidase (HRP)-conjugated secondary antibodies were purchased from Millipore.

Small-interfering RNA transfection for LC3B knockdown. LC3B target-specific small-interfering RNA (siRNA) plasmid vector and a scrambled siRNA control plasmid were purchased from Shanghai GenePharma, Ltd. (Shanghai, China). The siRNA sequences used were: LC3B, sense: 5'-GCGAGU UGGUCA AGAUCAUTT-3' and antisense: 5'-AUGAUC UUGACCAACUCGCTT-3'; negative control, sense: 5'-UUC UCCGAACGUGUCACGUTT-3' and antisense: 5'-ACGUGA CACGUUCGGAGAATT-3'. The cells were transfected with siRNA using Lipofectamine 2000 (Invitrogen/Life Technologies) according to the manufacturer's instructions. Cells $\left(2 \times 10^{5}\right.$ per well) were seeded in 6-well culture plates at $30-50 \%$ confluence. Each plate was transfected with 100 pmol plasmid containing LC3B siRNA or negative control siRNA and $5 \mu 1$ Lipofectamine 2000. Transfection efficiency averaged $60-70 \%$ as measured by green fluorescent protein expression. The cells were allowed to recover in RPMI-1640 medium for $6 \mathrm{~h}$ after transfection. Western blotting was performed to validate the knockdown efficiency, and the cells were divided for different assays.

Induction of hypoxia. Hypoxic environmental conditions were achieved by culturing cells in an airtight humidified chamber with a gas mixture containing $1 \% \mathrm{O}_{2}, 5 \% \mathrm{CO}_{2}$ and $94 \% \mathrm{~N}_{2}$. After $48 \mathrm{~h}$, transfection with siRNA was performed or 3-MA was dissolved in phosphate-buffered saline, pH 7.4 (PBS) and added to the medium at a final concentration of $1.25,2.5$, or $5 \mathrm{mM}$. The flasks were exposed to hypoxic conditions or maintained in a normoxic environment for an additional $24 \mathrm{~h}$, and the assays were performed.

Western blotting. Cells were collected for western blotting to determine the protein expression and trypan blue exclusion to determine cell viability. Briefly, proteins from the total cell lysates were separated by $10-15 \%$ sodium dodecyl sulfate polyacrylamide gel electrophoresis (SDS-PAGE) and then transferred to a nitrocellulose membrane (Bio-Rad Laboratories, Hercules, CA, USA). The membrane was blocked in 5\% non-fat dried milk, washed in Tris-buffered saline containing $0.1 \%$ Tween-20, and incubated at $4{ }^{\circ} \mathrm{C}$ overnight with specific primary antibodies: Rabbit polyclonal anti-LC3B antibody (1:500 dilution), mouse monoclonal anti-RhoA antibody (1:1000 dilution), rabbit monoclonal anti-ROCK1 antibody (1:1000 dilution), and mouse monoclonal anti- $\beta$-actin antibody (1:5000 dilution) as a loading control. This was followed by incubation with HRP-conjugated secondary antibodies. The proteins were detected with an enhanced chemiluminescence detection system (GE Healthcare, Little Chalfont, Buckinghamshire, UK).

Cell migration, invasion and adhesion assays. Cell migration was assessed with a Transwell chamber system (Corning Inc., Life Sciences, Tewksbury, MA, USA). A total of $2.0 \times 10^{5}$ cells in RPMI-1640 medium were added to the upper chamber under normoxic or hypoxic conditions. RPMI-1640 medium containing 
A
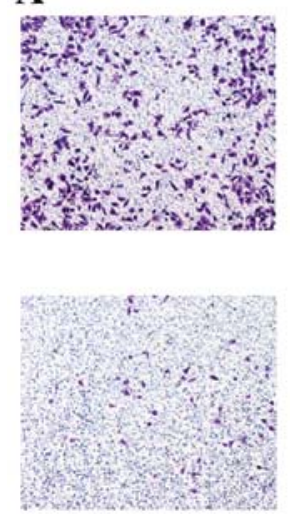

Normoxic

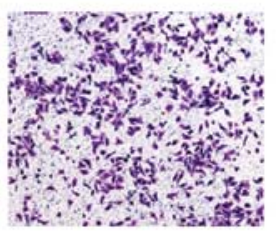

HO8910PM
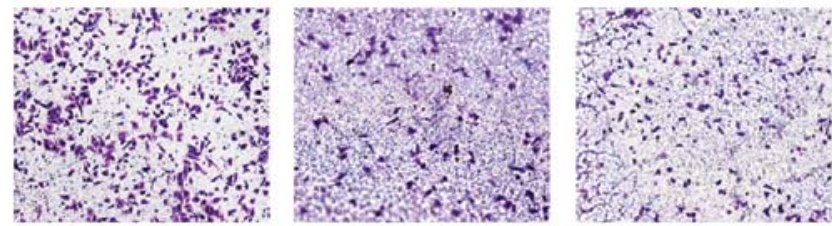

HO8910

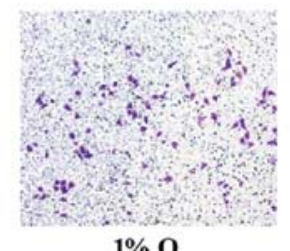

$1 \% \mathrm{O}_{2}$
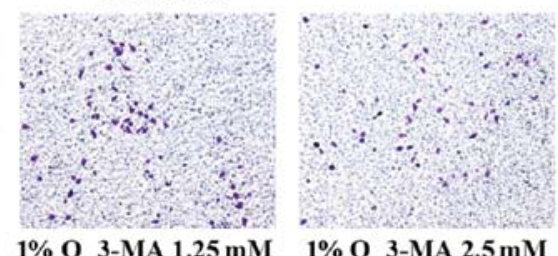

$1 \% \mathrm{O}_{2}$ 3-MA $2.5 \mathrm{mM}$

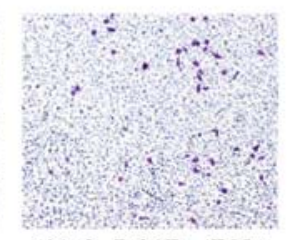

$1 \% \mathrm{O}_{2}$ LC3B-siRNA

B

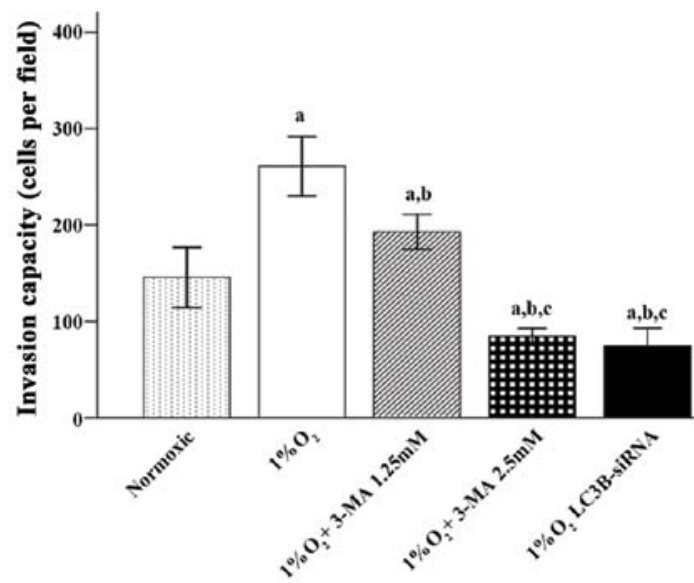

C

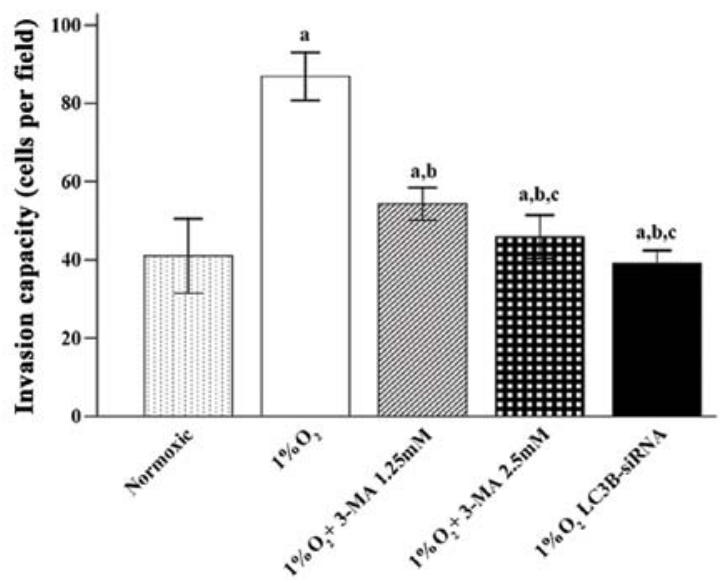

Figure 1. Epithelial ovarian cancer (EOC) cell migration following exposure to hypoxic conditions for $6 \mathrm{~h}$. (A) Representative images. (B) HO8910PM cells. (C) HO8910 cells. Data are presented as mean \pm standard deviation. The letter (a) denotes a significant difference compared to normoxic cells, (b) a significant difference compared to control cells ( $1 \% \mathrm{O}_{2}$ /hypoxia alone), and (c) a significant difference compared to 1.25 mM 3-methyladenine (3-MA)-treated cells $(\mathrm{p}<0.05)$. OD, optical density; siRNA, small-interfering RNA.

$10 \%$ FBS was added to the lower chamber. Migration assays were conducted for $6 \mathrm{~h}$ at $37^{\circ} \mathrm{C}$. The insert was then washed with 1X PBS, and the non-migrated cells were removed with a cotton swab. The cells were fixed in $4 \%$ paraformaldehyde and stained with $0.1 \%$ crystal violet. Excess staining was washed away with water. The cells were identified under a microscope, and a cell count of five different fields was performed.

Cell invasion was assessed with Matrigel-coated 24-well chambers. A total of $1.0 \times 10^{5}$ cells in RPMI-1640 medium were added to the insert. RPMI-1640 medium containing $10 \%$ FBS was added to the lower chamber. Invasion assays were conducted for $24 \mathrm{~h}$ at $37^{\circ} \mathrm{C}$ under normoxic or hypoxic conditions, and the cells were fixed and counted as described for cell migration assays. For the invasion assays involving siRNA, the cells were transfected with LC3B siRNA for $48 \mathrm{~h}$ prior to invasion assays being performed.

Cell adhesion was assessed with Matrigel-coated 96-well plates [dry-coated with Matrigel and blocked with $1 \%$ bovine serum albumin (BSA) for $1 \mathrm{~h}$ ]. A total of $4 \times 10^{4}$ cells were allowed to adhere to Matrigel-coated wells for $2 \mathrm{~h}$ at $37^{\circ} \mathrm{C}$ under hypoxic conditions. The wells were washed three times with PBS. A volume of $10 \mu 1$ 3-(4,5-dimethylthiazol-2-yl)-2,5-diphenyltetrazolium bromide (MTT) substrate was added to each well, and incubation was continued for an additional $4 \mathrm{~h}$. The number of adherent cells in each well was quantified by staining with dimethyl sulfoxide and absorbance was measured at $490 \mathrm{~nm}$.

Staining of cytoskeletal components. Control cells or cells subjected to LC3B knockdown were washed with PBS and blocked with $1 \%$ BSA. The cells were then incubated with rabbit polyclonal anti-LC3B antibody (1:500 dilution) at $4{ }^{\circ} \mathrm{C}$ overnight. The cells were washed with PBS, incubated with AlexaFluor 488 goat anti-rabbit $\operatorname{IgG}(\mathrm{H}+\mathrm{L})$ (1:300 dilution) for $2 \mathrm{~h}$, and rhodamine phalloidin (1:1000 dilution) was added for the last $30 \mathrm{~min}$. Cell nuclei were stained during the last $8 \mathrm{~min}$ with $20 \mu \mathrm{l}$ 4',6-diamidino-2-phenylindole (DAPI). Images were captured by laser confocal microscopy (Zeiss LSM 710), and image analysis was performed with ZEN microscopic image analysis software (Zeiss).

RhoA pull-down assay. Activated RhoA in cells was detected with Rho Assay Reagent according to the manufacturer's instructions. Activated GTP-RhoA was detected by western blotting and mouse monoclonal anti-RhoA antibody (1:1000 dilution). 
A
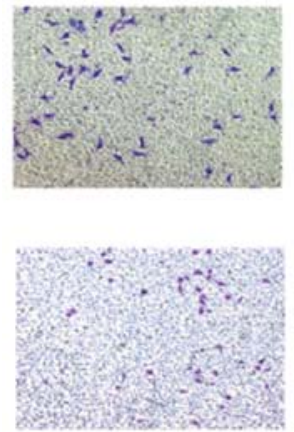

Normoxic

B

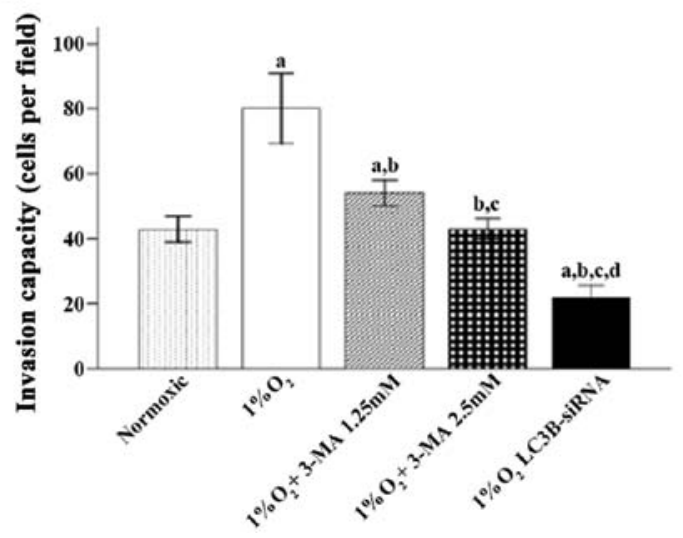

HO8910PM
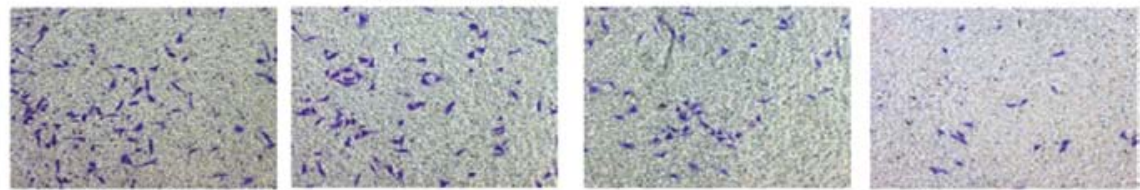

HO8910
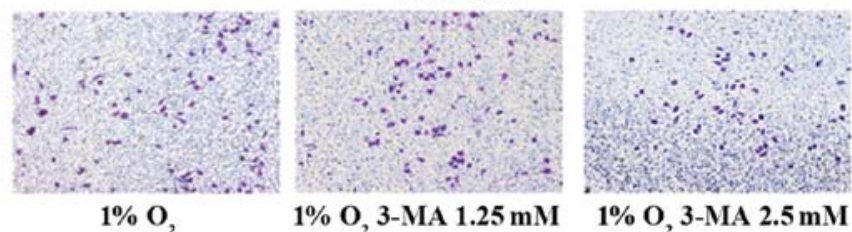

$1 \% \mathrm{O}_{2} 3-\mathrm{MA} 2.5 \mathrm{mM}$

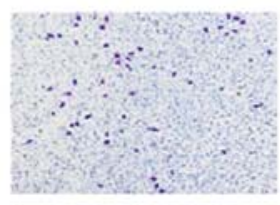

$1 \% \mathrm{O}_{2}$ LC3B-siRNA

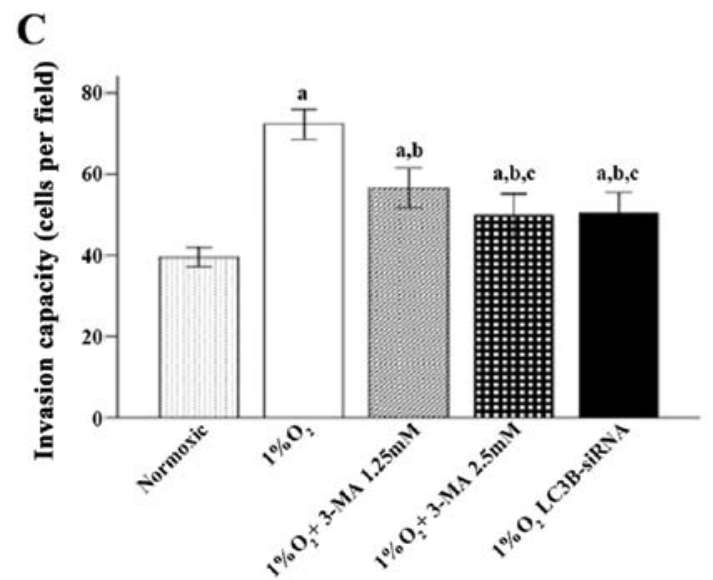

Figure 2. Epithelial ovarian cancer (EOC) cell invasion following exposure to hypoxic conditions for $24 \mathrm{~h}$. (A) Representative images. (B) HO8910PM cells. (C) HO8910 cells. Data are presented as mean \pm standard deviation. The letter (a) denotes a significant difference compared to normoxic cells, (b) a significant difference compared to control cells ( $1 \% \mathrm{O}_{2}$ /hypoxia alone), (c) a significant difference compared to $1.25 \mathrm{mM} 3$-methyladenine (3-MA)-treated cells, and (d) a significant difference from $2.5 \mathrm{mM} 3$-MA-treated cells ( $\mathrm{p}<0.05$ ). OD, optical density; siRNA, small-interfering RNA.
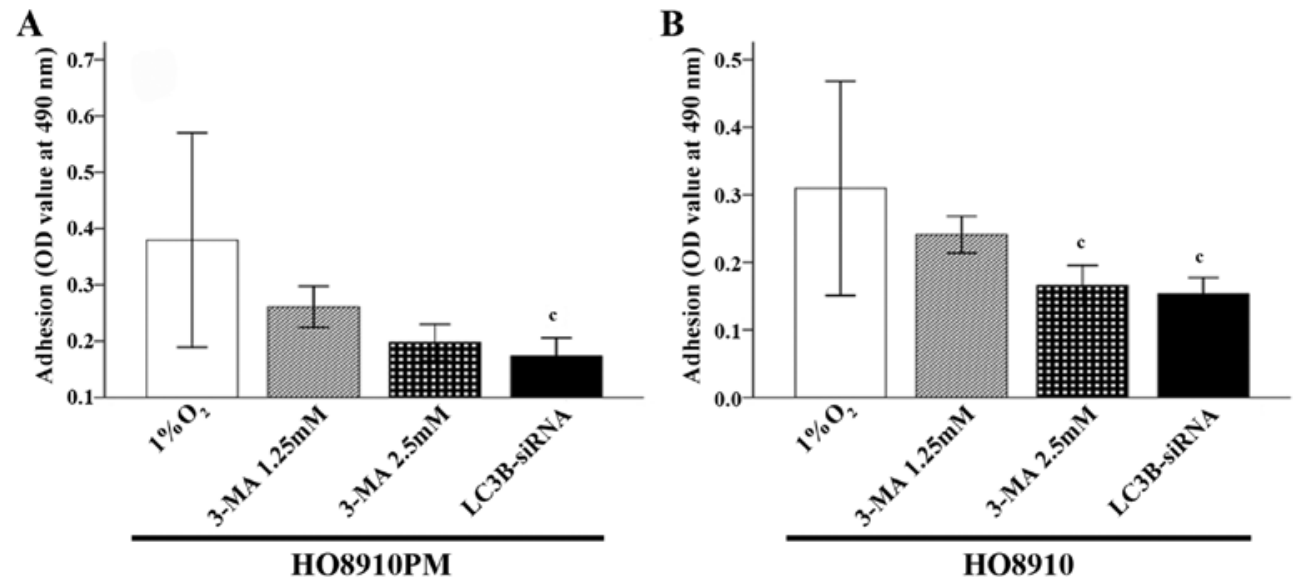

Figure 3. Epithelial ovarian cancer (EOC) cell adhesion following exposure to hypoxic conditions for 6 h. (A) HO8910PM cells. (B) HO8910 cells. Data are presented as mean \pm standard deviation. The letter (c) indicates a significant difference compared to $1.25 \mathrm{mM} 3$-methyladenine (3-MA)-treated cells ( $<<0.05)$. OD, optical density; siRNA, small-interfering RNA.

Statistical analysis. Experiments were repeated at least three times. Continuous data are presented as mean \pm standard deviation. To assess differences among groups, One-way analysis of variance was performed, followed by the Tukey and Dunnett T3 post-hoc tests. $\mathrm{P}<0.05$ was considered statistically significant. All the statistical analyses were two-sided and performed with SPSS software (version 15.0; SPSS Inc., Chicago, IL, USA).

\section{Results}

EOC migration and invasion are decreased by $L C 3 B$ siRNA or 3-MA treatment under hypoxic conditions. The results showed that hypoxic conditions promoted HO8910PM and HO8910 cell migration, invasion and adhesion (Figs. 1-3, respectively). When LC3B was blocked with 3-MA or LC3B siRNA treatment, cell migration and invasion were significantly decreased 
A
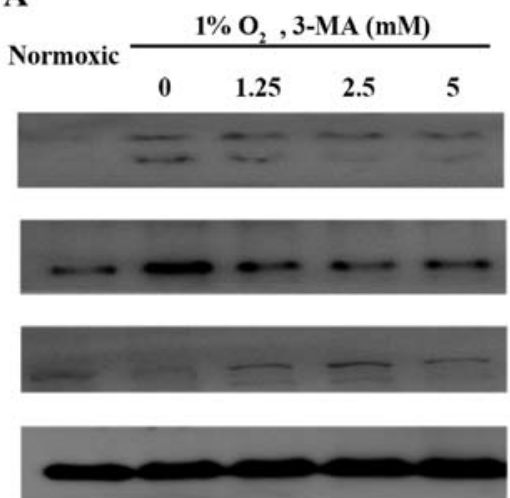

B
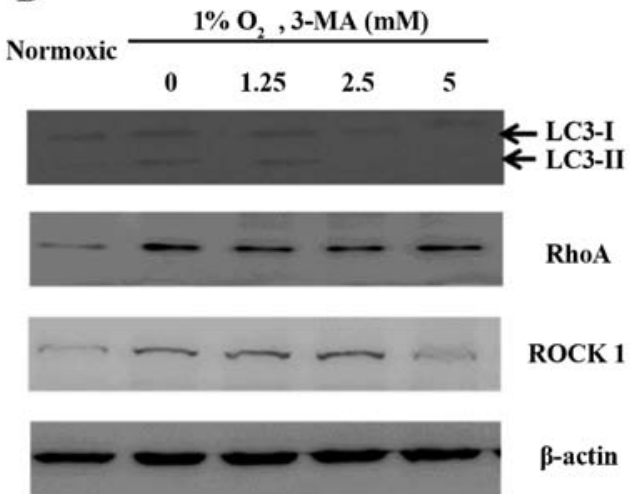

RhoA

ROCK 1

$\beta$-actin

Figure 4. Representative western blot data showing microtubule-associated protein 1 light chain 3B (LC3B), ras homolog gene family, member A (RhoA), and anti-Rho-associated, coiled-coil-containing protein kinase 1 (ROCK1) protein expression in (A) HO8910PM cells. (B) HO8910 cells under conditions of normoxia or under conditions of hypoxia and with or without treatment with increasing concentrations of 3-methyladenine (3-MA). $\beta$-actin was used as a loading control.
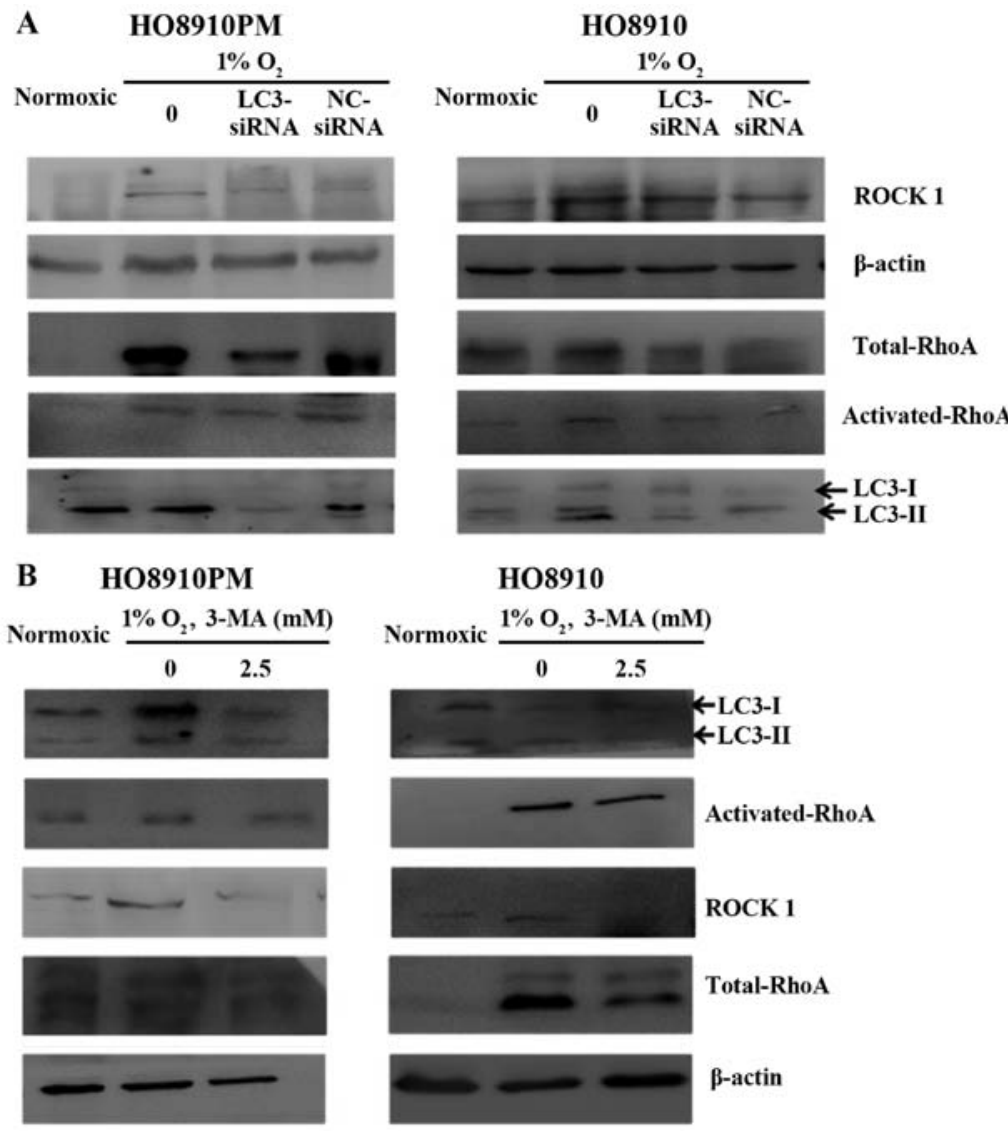

Figure 5. Representative western blot data showing microtubule-associated protein 1 light chain 3B (LC3B), ras homolog gene family member A (RhoA; activated and total), and anti-Rho-associated, coiled-coil-containing protein kinase 1 (ROCK1) protein expression in epithelial ovarian cancer (EOC) cell lines under conditions of normoxia or under conditions of hypoxia and (A) with or without LC3B small-interfering RNA (siRNA) [or normal control (NC) siRNA] or (B) 3-methyladenine (3-MA) treatment. $\beta$-actin was used as a loading control.

compared to that in control cells $\left(1 \% \mathrm{O}_{2}\right.$ /hypoxia alone; all $\mathrm{p}<0.001$ for both HO8910PM and HO8910 cells). In addition, the cells treated with LC3B siRNA showed significantly less migration ( $\mathrm{p}=0.021$ in HO8910PM cells and $\mathrm{p}<0.001$ in HO8910 cells) (Fig. 1) and invasion ( $p<0.001$ for the two cell types) (Fig. 2) compared to that in $1.25 \mathrm{mM} 3$-MA-treated cells. Adhesion was significantly reduced in cells treated with LC3B siRNA compared to that in $1.25 \mathrm{mM} 3$-MA-treated cells $(\mathrm{p}=0.021$ in HO8910PM cells and $\mathrm{p}=0.004$ in HO8910 cells) (Fig. 3).

Effect of LC3B siRNA or 3-MA treatment on LC3B and RhoA expression in HO8910PM and HO8910 cells under hypoxic conditions. LC3B expression and conversion of LC3B-I to LC3B-II was observed in the two cell lines under hypoxic conditions (Figs. 4 and 5). In addition, RhoA expression was clearly 

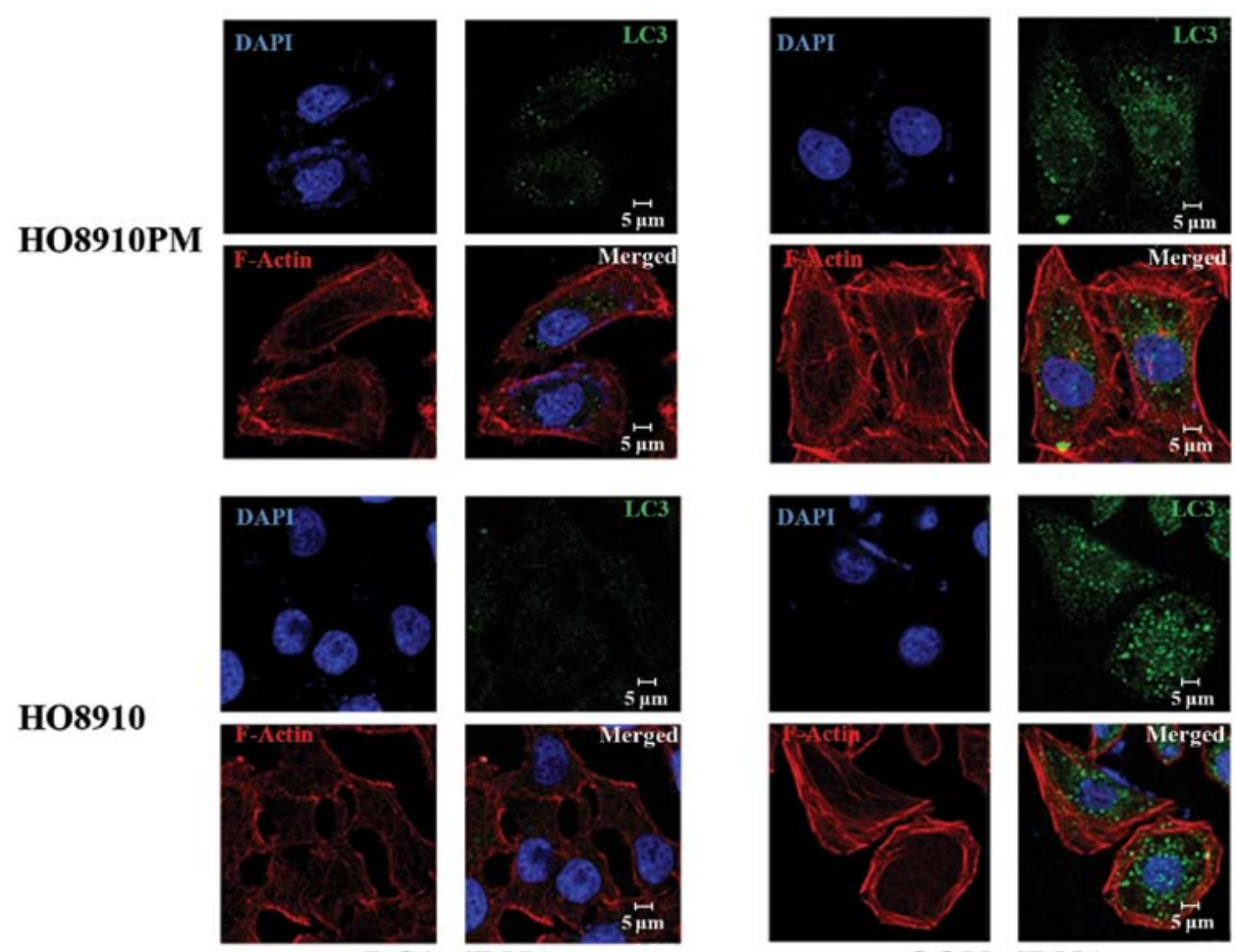

LC3 siRNA

CON SiRNA

Figure 6. HO8910PM and HO8910 cells transfected with microtubule-associated protein 1 light chain 3B small interfering RNA (LC3 siRNA) or control siRNA (CON siRNA) were maintained under hypoxic conditions for $24 \mathrm{~h}$ and visualized by confocal microscopy after costaining with anti-LC3B antibody (green), rhodamine phalloidin for filamentous actin (F-actin; red), and the nuclear dye 4',6-diamidino-2-phenylindole (DAPI; blue).

increased under hypoxic conditions. LC3B siRNA (Fig. 5) and 24-h 3-MA treatment (Figs. 4 and 5) each decreased LC3B expression under hypoxic conditions. Control siRNA had no effect on LC3B expression. We also found that 3-MA treatment decreased activated RhoA expression and expression of the downstream effector molecule ROCK1 in HO8910 cells, and LC3B siRNA decreased activated RhoA and ROCK1 expression in HO8910PM cells. We also confirmed hypoxia-induced RhoA expression, which was inhibited via LC3B siRNA in the two cell lines, though it appeared that control siRNA had some interference effect on RhoA expression in HO8910 cells. These results suggested that RhoA may be associated with $\mathrm{LC} 3 \mathrm{~B}$ and that the mechanism by which LC3B promotes metastasis may involve the RhoA pathway in EOC cells.

Effect of LC3B siRNA on filamentous actin. Filamentous actin (F-actin) and LC3B in EOCs under hypoxic conditions were detected by laser scanning confocal microscopy (Fig. 6). The cells transfected with LC3 siRNA showed F-actin fibers that lacked tension and direction and showed a disordered arrangement. The cells transfected with control siRNA showed F-actin fibers with good tension and direction and filament bundles, suggestive of good invasion ability.

\section{Discussion}

The aim of the present study was to determine whether LC3B is involved in the migration and invasion of EOC cells. We hypothesized that LC3B expression is advantageous for tumor development and that inhibition of LC3B is potentially effective in the treatment or prevention of metastasis, potentially by

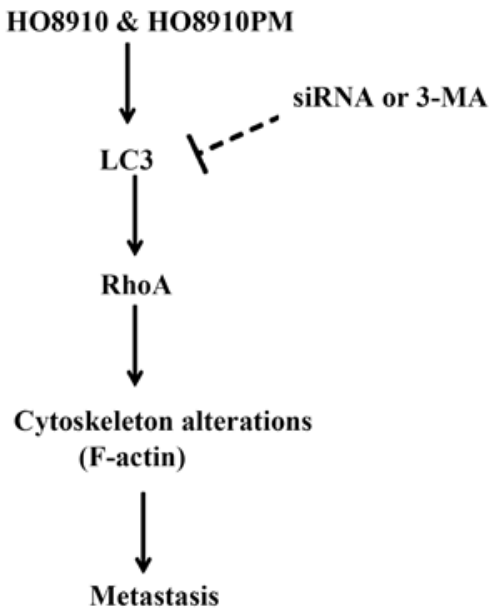

Figure 7. Schematic diagram showing a potential mechanism whereby microtubule-associated protein 1 light chain 3B (LC3) promotes the migration and invasion of epithelial ovarian cancer cells, involving the ras homolog gene family member A (RhoA) pathway and thus affecting the cytoskeleton. siRNA, small-interfering RNA; 3-MA, 3-methyladenine; F-actin, filamentous actin.

depriving tumor cells of sources of energy. Using an in vitro Transwell migration assay, we showed that inhibition of LC3B via LC3B siRNA or 3-MA treatment decreased EOC cell migration and invasion.

HO8910PM and HO8910 EOC cells showed a weak LC3B expression under normoxic conditions and increased LC3B expression under hypoxic conditions. Inhibition of LC3B expression via siRNA or 24-h 3-MA treatment reduced the expression of hypoxia-induced LC3B to levels similar to those shown under 
normoxic conditions. These results support the potential utility of LC3B as an endogenous marker of tumor hypoxia.

Notably, the downregulation of LC3B decreased the expression of RhoA and its downstream effector ROCK1, suggesting an association between hypoxia-induced LC3B and RhoA. The cytoskeleton is important for maintaining cell shape for motility. Members of the Rho GTPase family regulate the organization and stability of F-actin including in actin cytoskeleton remodeling (membrane protrusion, cell adhesion, and motility) (18). Cell protrusion and spreading are associated with integrin-matrix interactions for surface attachment and focal adhesion function (30). RhoA regulates the formation of stress fibers and the contractile ring via the stimulation of actin polymerization and activation of myosin (18). LC3B siRNA-transfected cells showed broken and disorganized F-actin fibers, with a lack of tension and direction. This was associated with reduced cell migration and invasion. LC3B siRNA thus had an effect on actin filaments, potentially by modulating RhoA activity (Fig. 7). The specific mechanism(s) whereby LC3B affects RhoA GTPase activity and leads to changes in the cytoskeleton remains to be elucidated.

In conclusion, the present results emphasize the potential of LC3 as a marker of hypoxia and/or metastasis and demonstrate that the inhibition of LC3 may be a promising strategy for reducing metastasis of human malignant EOC cells. In vivo studies may prove useful in assessing this potential.

\section{Acknowledgements}

We thank Dr Yi Jing (Department of Biochemistry and Molecular Cell Biology, Shanghai Jiao Tong University School of Medicine, Shanghai, China) for technical assistance. This study was supported by the National Natural Science Foundation of China (grant no. 81101972).

\section{References}

1. Davidson B, Reich R, Trope CG, Wang TL and Shih IeM: New determinates of disease progression and outcome in metastatic ovarian carcinoma. Histol Histopathol 25: 1591-1609, 2010.

2. Bagnato A, Spinella F and Rosanò L: Emerging role of the endothelin axis in ovarian tumor progression. Endocr Relat Cancer 12: 761-772, 2005

3. Itamochi H: Targeted therapies in epithelial ovarian cancer: molecular mechanisms of action. World J Biol Chem 1: 209-220, 2010.

4. Opipari AW Jr, Tan L, Boitano AE, Sorenson DR, Aurora A and Liu JR: Resveratrol-induced autophagocytosis in ovarian cancer cells. Cancer Res 64: 696-703, 2004.

5. Simonin K, Brotin E, Dufort S, Dutoit S, Goux D, N'diaye M, Denoyelle C, Gauduchon P and Poulain L: Mcl-1 is an important determinant of the apoptotic response to the $\mathrm{BH} 3$-mimetic molecule HA14-1 in cisplatin-resistant ovarian carcinoma cells Mol Cancer Ther 8: 3162-3170, 2009.

6. Saad AF, Hu W and Sood AK: Microenvironment and pathogenesis of epithelial ovarian cancer. Horm Cancer 1: 277-290, 2010.

7. Zhang J, Yang Z, Xie L, Xu L, Xu D and Liu X: Statins, autophagy and cancer metastasis. Int J Biochem Cell Biol 45: 745-752, 2013

8. Bhoopathi P, Gondi CS, Gujrati M, Dinh DH and Lakka SS: SPARC mediates Src-induced disruption of actin cytoskeleton via inactivation of small GTPases Rho-Rac-Cdc42. Cell Signal 23: 978-1987, 2011.

9. Du H, Yang W, Chen L, Shen B, Peng C, Li H, Ann DK, Yen Y and Qiu W: Emerging role of autophagy during ischemia-hypoxia and reperfusion in hepatocellular carcinoma. Int J Oncol 40: 2049-2057, 2012.
10. Sun Y, Liu JH, Sui YX, Jin L, Yang Y, Lin SM and Shi H: Beclin1 overexpression inhibits proliferation, invasion and migration of CaSki cervical cancer cells. Asian Pac J Cancer Prev 12: 1269-1273, 2011

11. Rouschop KM and Wouters BG: Regulation of autophagy through multiple independent hypoxic signaling pathways. Curr Mol Med 9: 417-424, 2009.

12. Schlie K, Spowart JE, Hughson LR, Townsend KN and Lum JJ: When cells suffocate: autophagy in cancer and immune cells under low oxygen. Int J Cell Biol 2011: 470-597, 2011.

13. Indelicato M, Pucci B, Schito L, Reali V, Aventaggiato M, Mazzarino MC, Stivala F, Fini M, Russo MA and Tafani M: Role of hypoxia and autophagy in MDA-MB-231 invasiveness. J Cell Physiol 223: 359-368, 2010.

14. Tanida I, Ueno T and Kominami E: LC3 conjugation system in mammalian autophagy. Int J Biochem Cell Biol 36: 2503-2518, 2004.

15. Martinet W, De Meyer GR, Andries L, Herman AG and Kockx MM: In situ detection of starvation-induced autophagy. J Histochem Cytochem 54: 85-96, 2006.

16. Peracchio $\mathrm{C}$, Alabiso $\mathrm{O}$, Valente $\mathrm{G}$ and Isidoro $\mathrm{C}$ : Involvement of autophagy in ovarian cancer: a working hypothesis. J Ovarian Res 5: 22, 2012.

17. Hu YL, DeLay M, Jahangiri A, Molinaro AM, Rose SD, Carbonell WS and Aghi MK: Hypoxia-induced autophagy promotes tumor cell survival and adaptation to antiangiogenic treatment in glioblastoma. Cancer Res 72: 1773-1783, 2012.

18. Aguilera MO, Berón W and Colombo MI: The actin cytoskeleton participates in the early events of autophagosome formation upon starvation induced autophagy. Autophagy 8: 1590-1603, 2012.

19. Pankiv S, Alemu EA, Brech A, Bruun JA, Lamark T, Overvatn A, Bjørkøy G and Johansen T: FYCO1 is a Rab7 effector that binds to LC3 and PI3P to mediate microtubule plus end-directed vesicle transport. J Cell Biol 188: 253-269, 2010.

20. Rapisarda A,Uranchimeg B, Scudiero DA, Selby M, Sausville EA, Shoemaker RH and Melillo G: Identification of small molecule inhibitors of hypoxia-inducible factor 1 transcriptional activation pathway. Cancer Res 62: 4316-324, 2002.

21. Lazova R, Camp RL, Klump V, Siddiqui SF, Amaravadi RK and Pawelek JM: Punctate LC3B expression is a common feature of solid tumors and associated with proliferation, metastasis, and poor outcome. Clin Cancer Res 18: 370-379, 2012.

22. Sato K, Tsuchihara K, Fujii S, Sugiyama M, Goya T, Atomi Y, Ueno T, Ochiai A and Esumi H: Autophagy is activated in colorectal cancer cells and contributes to the tolerance to nutrient deprivation. Cancer Res 67: 9677-9684, 2007.

23. Yoshioka A, Miyata H, Doki Y, Yamasaki M, Sohma I, Gotoh K, Takiguchi S, Fujiwara Y, Uchiyama Y and Monden M: LC3, an autophagosome marker, is highly expressed in gastrointestinal cancers. Int J Oncol 33: 461-468, 2008.

24. Han C, Sun B, Wang W, Cai W, Lou D, Sun Y and Zhao X: Overexpression of microtubule-associated protein-1 light chain 3 is associated with melanoma metastasis and vasculogenic mimicry. Tohoku J Exp Med 223: 243-251, 2011.

25. Fujii S, Mitsunaga S, Yamazaki M, Hasebe T, Ishii G, Kojima M, Kinoshita T, Ueno T, Esumi $\mathrm{H}$ and Ochiai A: Autophagy is activated in pancreatic cancer cells and correlates with poor patient outcome. Cancer Sci 99: 1813-1819, 2008.

26. Qin AP, Liu CF, Qin YY, Hong LZ, Xu M, Yang L, Liu J, Qin ZH and Zhang HL: Autophagy was activated in injured astrocytes and mildly decreased cell survival following glucose and oxygen deprivation and focal cerebral ischemia. Autophagy 6: 738-753, 2010.

27. Codogno P and Meijer AJ: Autophagy and signaling: their role in cell survival and cell death. Cell Death Differ 12 (Suppl 2): 1509-1518, 2005

28. Myeku N and Figueiredo-Pereira ME: Dynamics of the degradation of ubiquitinated proteins by proteasomes and autophagy: association with sequestosome $1 / \mathrm{p} 62$. J Biol Chem 286: 22426-22440, 2011

29. Song J, Qu Z, Guo X, Zhao Q, Zhao X, Gao L, Sun K, Shen F, Wu M and Wei L: Hypoxia-induced autophagy contributes to the chemoresistance of hepatocellular carcinoma cells. Autophagy 5: 1131-1144, 2009.

30. Kadandale P, Stender JD, Glass CK and Kiger AA: Conserved role for autophagy in Rhol-mediated cortical remodeling and blood cell recruitment. Proc Natl Acad Sci USA 107: 10502-10507, 2010. 